Reprod. Nutr. Dévelop., 1980, 20 (4 B), 1193-1196.

\title{
Corrélations électromyographiques des mouvements des ingesta dans les segments digestifs proximaux chez la Poule
}

par M. ROCHE

Insfitut National des Sciences Appliquées de Lyon, Bât. 406,

20, avenue Albert-Einstein, 69621 Villeurbanne Cedex, France.

Summary. An electromyographic study of propulsion in the proximal parts of the digestive tract in the hen.

Digesta transit along the oesophagus, crop and stomach has been determined by the migration of spike bursts correlated with cineradiographic observations in hens fitted with chronicaliy implanted electrodes. The passage of digesta along the oesophagus corresponded to the propagation of isolated spike bursts. During crop filling, the activity was weak, its emptying being mediated by migrating repetitive spike bursts. A cyclically recurring electrical activity was noted for the glandular and muscular stomachs and the duodenum ; their motility is described in relation to digesta movements.

\section{Introduction.}

Chez les Oiseaux granivores, les mouvements des ingesta dans les segments digestifs proximaux sont conditionnés par trois particularités anatomo-fonctionnelles : (i) dilatation de l'œsophage en un jabot, (ii) différenciation du territoire gastrique en estomacs glandulaire et musculaire, (iii) abouchement des conduits biliaires à la terminaison du duodénum. Des études électromyographiques antérieures ayant pour but de définir chez le Poulet le profil moteur du tractus intestinal nous ont permis de souligner le caractère univoque de l'activité électrique (salves de potentiels de pointe) (Roche, 1974) et de démontrer d'une part l'indépendance relative des muscles supérieurs et inférieurs constituant les mâchoires de l'estomac musculaire (Roche, 1973), d'autre part l'importance de la propagation antidromique des salves de potentiels le long de l'intestin (Roche et Ruckebusch, 1978).

La présente étude, réalisée chez la Poule, est relative à l'enregistrement de l'activité électrique globale de la paroi musculaire des territoires prégastriques (œsophage supérieur et jabot) et gastrique (estomacs glandulaire et musculaire) en fonction du transit des ingesta.

\section{Techniques expérimentales et matériel utilisés.}

4 Poules élevées dans des cages individuelles permettant des enregistrements continus pendant 8 semaines ont été utilisées. Elles sont soumises à un éclairement standard de $12 \mathrm{~h} / 24$ et disposent d'une alimentation complète présentée sous forme de 
farine. L'électromyogramme (EMG) est enregistré à partir d'électrodes au $\mathrm{NiCr}$ (diamètre $100 \mathrm{~nm}$ et longueur $80 \mathrm{~cm}$ ), implanfées par paire selon une technique déjà décrite (Roche et Ruckebusch, 1978). Pour chaque animal, 11 niveaux d'enregistrement différents ont été retenus à savoir : trois pour l'œsophage à 5,3 et $1 \mathrm{~cm}$ du jabot, trois pour le jabot à $1,2,5 \mathrm{~cm}$ de son pôle oral, une pour l'estomac glandulaire à proximité de la jonction avec l'estomac musculaire ef pour l'estomac musculaire, 4 électrodes réparties comme indiquées dans la figure 2.

Les enregistrements sont réalisés en réception bipolaire à l'aide d'un polygraphe Alvar XV pistes (constante de temps $0,1 \mathrm{sec}$.). L'électromyogramme est analysé en relation avec le transit des ingesta observé par cinéradiographie (Siemens) à partir soit de l'alimentation habituelle additionnée de 30 p. 100 de baryte soit d'une solution baryłée épaisse introduite directement dans l'estomac glandulaire.

\section{Résultałs.}

1) Terrifoire œsophageo-ingluvial. - La figure 1 présente les différents types d'activité électrique recueillis lors de l'ingestion alimentaire. Au niveau de l'œsophage le passage effectif des aliments solides résulte de la propagation (vitesse 0,8 à $1,2 \mathrm{~cm} / \mathrm{sec}$.), en direction du jabot, de salves de potentiels de pointe (durée $6 \mathrm{sec}$. ; amplitude 350 à $450 \mu v$ ). Une alimentation fournie sous forme de farine doit atteindre un volume seuil capable de déclencher la motricité œsophagienne : il en résulte que l'induction des salves de potentiels de pointe peut être précédée de 3 à 4 prises alimentaires. Par contre, le transit de l'eau est immédiat et correspond à la propagation rapide (5 à $7 \mathrm{~cm} / \mathrm{sec}$.) de courtes (1 à $2 \mathrm{sec}$.) salves de potentiels isodynames des précédentes.

PERIODE DIURNE

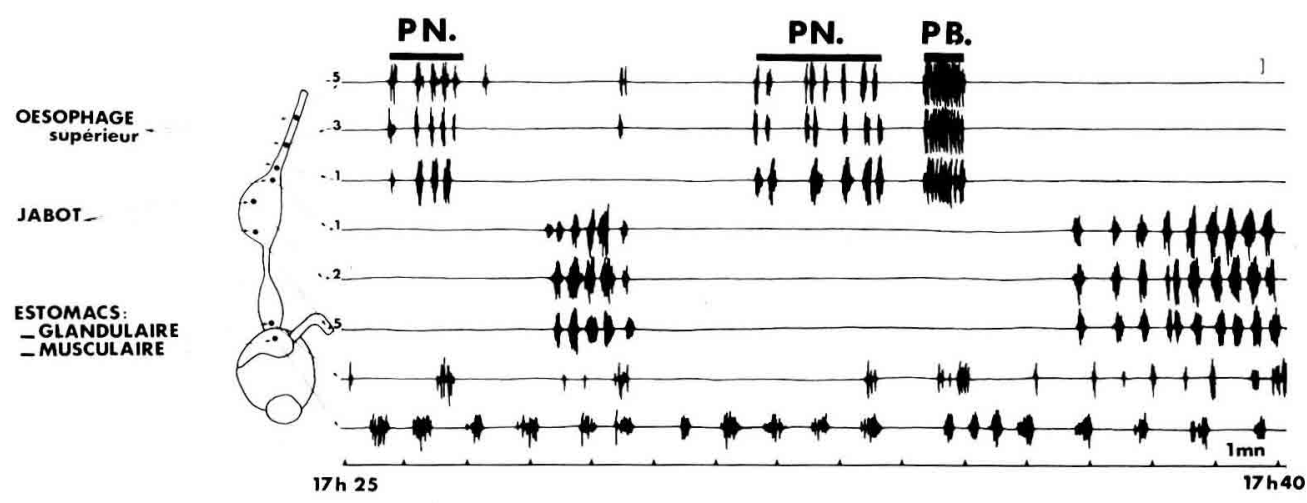

FIG. 1. - Propagation des bouffées de potentiels sur l'œesophage et le jabot en fonction du comportement alimentaire. Au niveau œsophagien, chaque groupe de salves de potentiels correspond au transit des ingesta. Noter l'inhibition du jabot lors de la prise de nourriture (PN) et de boisson (PB) (calibrage $100 \mu v$ ).

L'électromyogramme de la paroi musculaire du jabot est constitué de longues $(10-12 \mathrm{sec}$.) salves de potentiels d'amplitude comprise entre 250 et $450 \mu \mathrm{v}$. Au cours des périodes de prise de nourriture ou de boisson puis du transit des ingesta le long 
de l'œsophage supérieur, le jabot est électriquement silencieux. Des salves isolées ou regroupées par 3 à 5 assurent le brassage du contenu ingluvial par leur propagation ortho- ou antidromique. L'analyse de la répartition nycthémérale de l'activité électrique du jabot montre que sa dilatation par stockage des ingesta observée quelques heures avant le début de l'obscurité se traduit par une réduction progressive de la fréquence des salves de potentiels puis leur inhibition totale 30 à 45 min après la fin de l'éclairement. Lors de la vidange du jabot la propulsion progressive de son contenu en direction des territoires gastriques résulte de la propagation orthodromique de 10 à 20 salves de potentiels successives.

2) Territoires gastriques. - L'analyse simultanée de l'électromyogramme des différents territoires gastriques et des mouvements effectifs du contenu digestif a permis de différencier plusieurs modalités d'activation de l'électromyogramme, présentées dans la figure 2. Au cours de la phase diurne, l'estomac musculaire se contracte selon un rythme moyen de $1,9 / \mathrm{min}$. En absence de tout contenu digestif, l'activité électrique de l'estomac glandulaire se limite à une brève salve de potentieis intercallée entre l'activation des muscles intermédiaires et des mâchoires de l'estomac musculaire (fig. 2A). La prise de nourriture et de boisson modifie cette motricité sans perturber profondément son caractère cyclique. Ainsi la pénétration d'un bol alimentaire dans l'estomac glandulaire nécessite que celui-ci soit dilaté et électriquement silencieux ; elle est suivie d'une contraction de sa jonction avec l'estomac musculaire (fig. 2B). Le transit estomac glandulaire-estomac musculaire se réalise pendant une
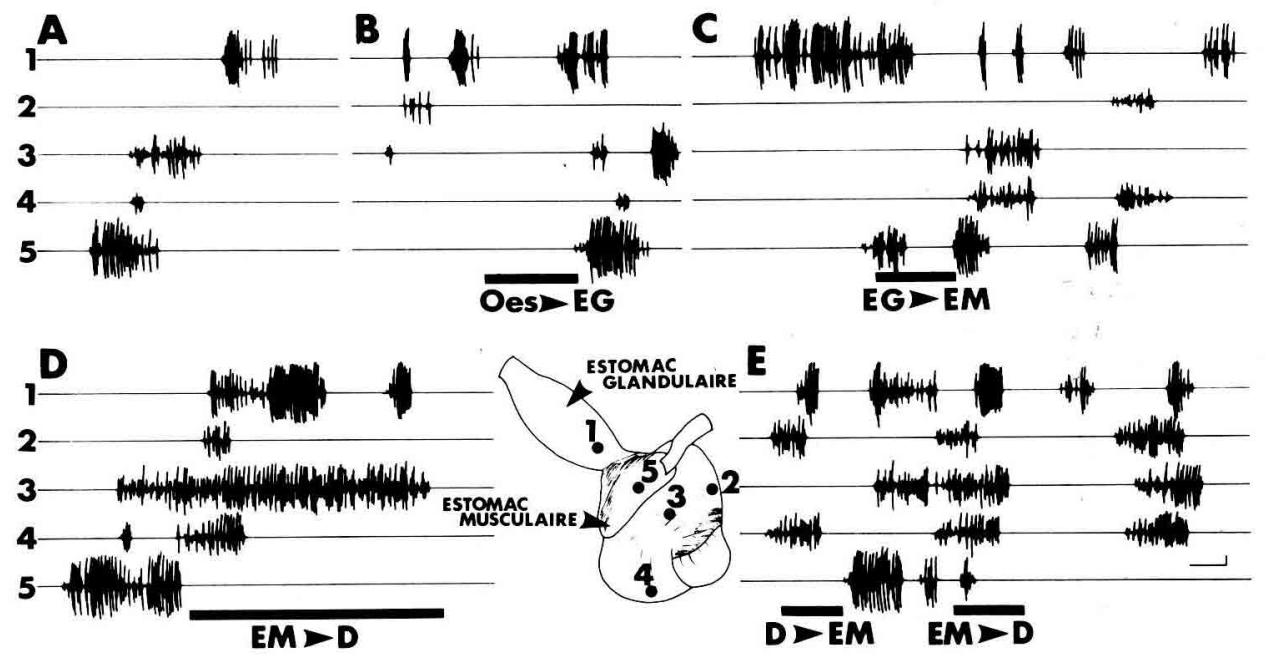

FIG. 2. - Modalités de coordination de l'activité des différents territoires gastriques en fonction du mouvement des ingesła. En A, à jeûn l'EMG des muscles intermédiaires (5) précède celui des mâchoires (3). En B, l'activation de l'estomac glandulaire précède et suit l'arrivée des ingesta dans ce dernier. En $C$, transit d'ingesta dans l'estomac musculaire par inhibition de ce dernier et contraction tonique de l'estomac glandulaire. En D, évacuation duodénale par contraction tonique de la zone médiane de la mâchoire (3), la contraction de la paroi de l'estomac glandulaire empêche le retour des ingesta vers ce dernier. En E, mouvements de va-et-vient entre le duodénum et l'estomac musculaire (œs., œsophage ; EG, estomac glandulaire ; EM, estomac musculaire ; D, Duodénum) (calibrage $100 \mu v$ et 6 sec.). 
inhibition de l'estomac musculaire sous l'influence d'une contraction soutenue (longue salve de potentiels) de l'estomac glandulaire (fig. 2C). L'évacuation des ingesta dans le duodénum résulte de la contraction soutenue de la région médiane des mâchoires (fig. 2D et E). La contraction de la parai de l'estomac glandulaire s'oppose à un reflux partiel du bol gastrique. Le reflux des ingesta vers l'estomac glandulaire est dû, lors d'un relâchement de celui-ci, à l'activation de l'ensemble de l'estomac musculaire.

\section{Discussion et conclusion.}

Bien que mal connues, la littérature accorde au jabot d'autres fonctions que celle de réservoir permettant de fragmenter le départ des ingesta vers les territoires gastriques : la production d'œufs peu calcifiés, voire sans coquille, après jabectomie va dans ce sens. En particulier, le jabot intervient dans l'absorption de l'acide lactique et, par là même, le contrôle de la sécrétion d'HCl par l'intermédiaire de la dissolution du calcium (Mongin, 1976). Il apparaît donc d'un particulier intérêt de connaître les mécanismes de remplissage et de vidange du jabot en fonction du nycthémère, de la ponte ou de la composition physique et chimique de la ration alimentaire. Les premiers résultats présentés à partir de notre étude électromyographique chronique démontre l'inhibition progressive de la motricité ingluviale lors du remplissage observé avant la période obscure du nycthémère. Quant aux territoires gastriques, la situation paradoxale de l'crgane broyeur, l'estomac musculaire, par rapport aux lieux de libération des enzymes, estomac glandulaire ef duodénum (Chawan, Bird et Gerry, 1977) nécessite des mouvements de va et vient du contenu digestif entre ces trois segments digestifs.

Journées Ingestion-Digestion-Absorption de l'Association française de Nutrition, Paris, 15-16 novembre 1979.

\section{Références}

CHAWAN C. B., BIRD F. H., GERRY R. W., 1977. Relationship between intestinal movements and pancreatic juice secretion in the domestic chicken. Poultry Sci., 57, 1084-1086.

MONGIN P., 1976. Composition of crop and gizzard contents in the laying hen. Br. Poult. Sci., 17, 499-507.

ROCHE M., 1973. Motricité digestive des oiseaux. Ełude électromyographique chez le Poulet (Gallus Gallus). Cah. Med. vét., 42, 259-271.

ROCHE M., 1974. Motricité gastro-intestinale du poulet. Ann. Rech. vét., 5, 295-309.

ROCHE M., RUCKEBUSCH Y., 1978. A basic relationships between gastric and duodenal motilities in chicken. Am. J. Physiol., 235, E670-E677. 